\title{
Transitivity markers in West Himalayish
}

\author{
$\operatorname{AUTHOR}(\mathrm{S})$ :
}

Widmer, Manuel

\section{CITATION:}

Widmer, Manuel. Transitivity markers in West Himalayish. Proceedings of the 51st International Conference on Sino-Tibetan Languages and Linguistics 2018: 66.

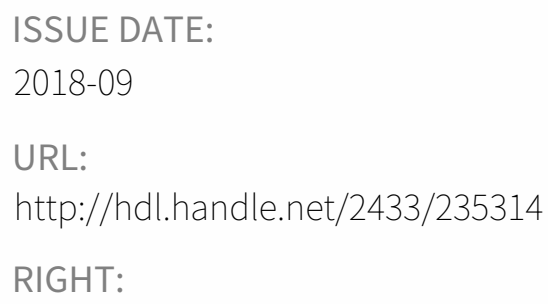




\section{Transitivity markers in West Himalayish}

ICSTLL 51, Kyoto University

Manuel Widmer, University of Zurich 


\section{The West Himalayish languages}




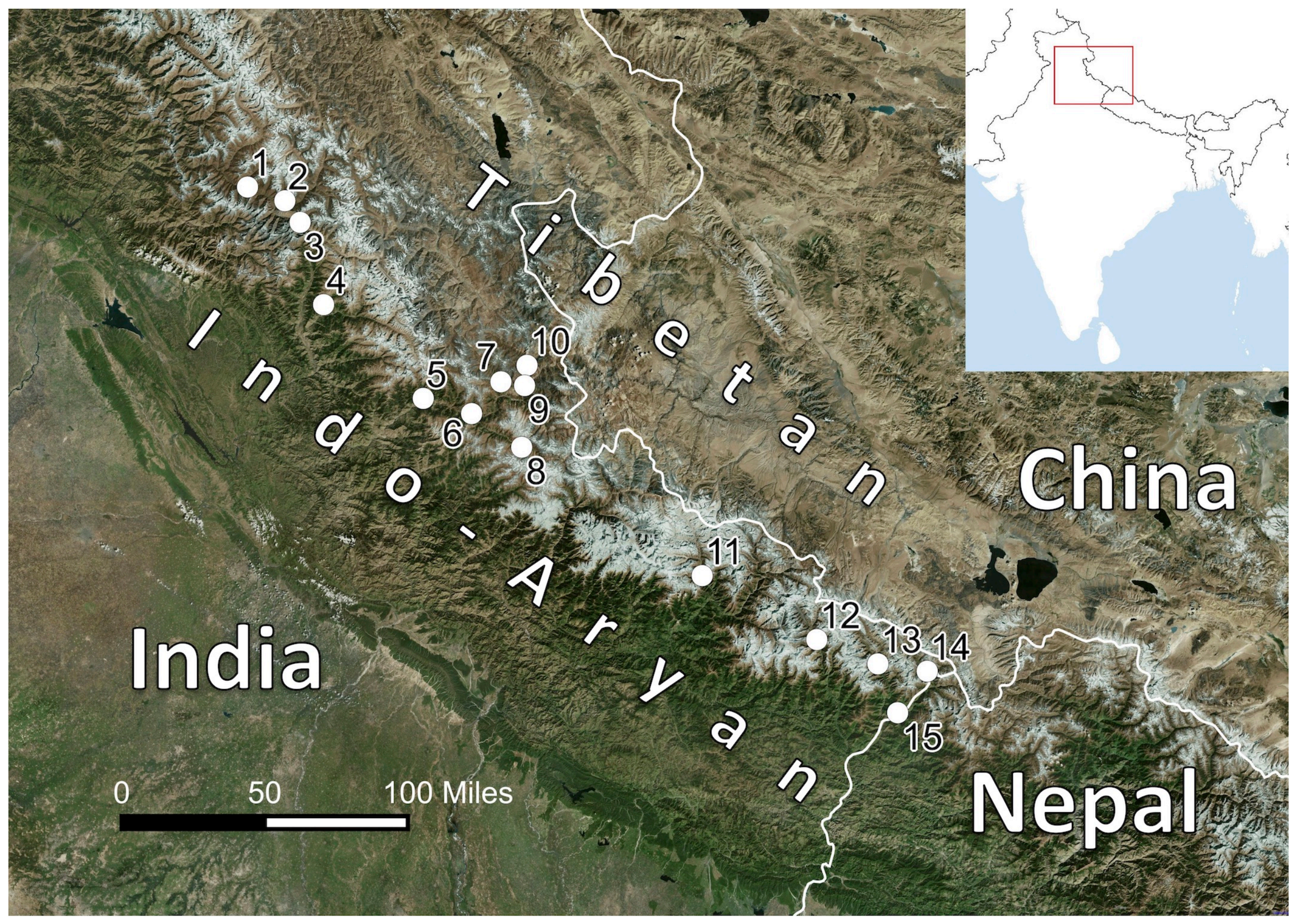




\section{Classifying West Himalayish}




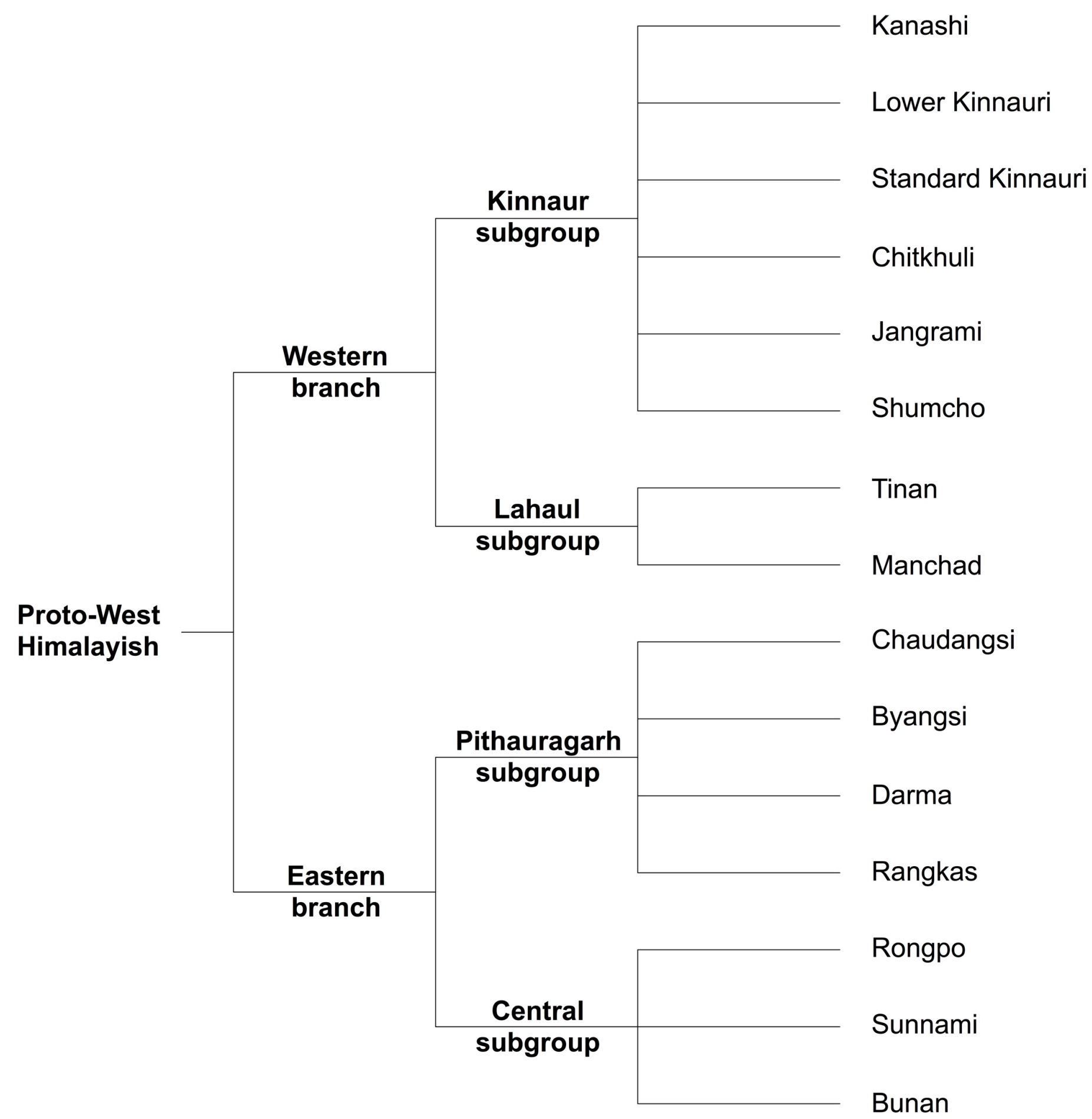




\section{Remarks on transitivity}




\section{Transitivity can be defined as a ...}

- Syntactic notion $\rightarrow$ classification of verbs into "intransitive" and "transitive" based on number of core arguments

(Dixon 2010)

- Semantic notion $\rightarrow$ classification of verbs into "intransitive" and "transitive" based on semantic properties

(Hopper \& Thompson 1980) 


\section{A working definition of transitivity}

- For this talk, the term "transitivity" is used to refer to a grammatical category that is marked on verbs and classifies them into transitivity classes. This classification can either be based on syntactic or semantic properties.

- The labels "intransitive" and "transitive", in turn, will be used to refer to verbal transitivity classes. 


\section{The term «valence»}

- The term "valence" will be used to describe how many core arguments a verb has (irrespective of its transitivity class membership)

- one core argument:

- two core arguments:

- three core arguments: monovalent

$\left.\begin{array}{l}\text { bivalent } \\ \text { trivalent }\end{array}\right\}$ plurivalent




\section{Transitivity classes in Bunan}




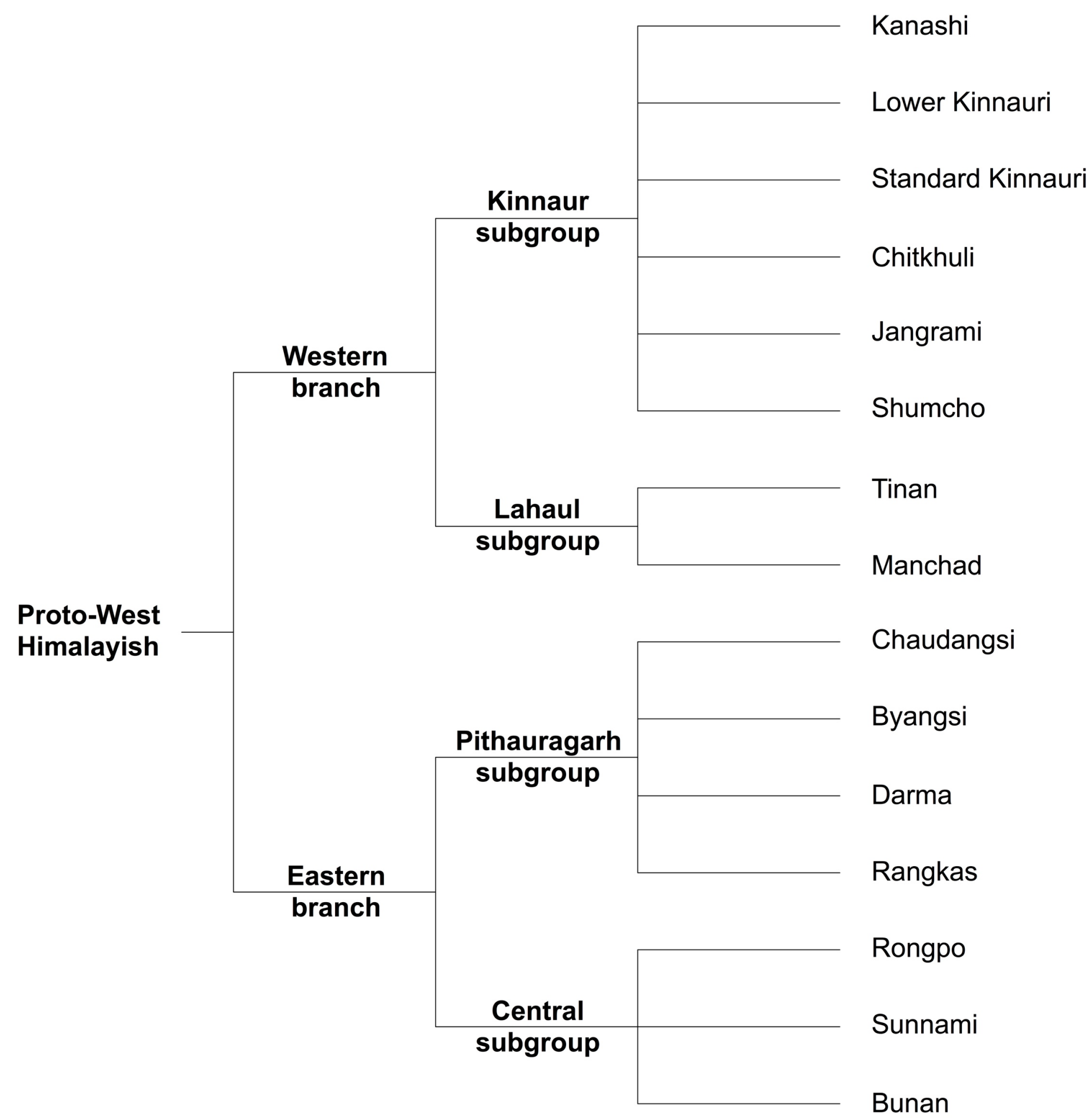




\section{Universität}

Zürich $^{\mathrm{UzH}}$

Department of Comparative Linguistics

\section{Transitivity classes in Bunan}

(1) a. Intransitive class: bjak-men 'to hide'

bjak-k-ek

hide-INTR-PRS.EGO.SG

'I am hiding myself.' (Widmer, fieldnotes)

b. Middle class: lok-6-um 'to climb up'

lok-6-ek

climb-MID-PRS.EGO.SG

'I am climbing up.' (Widmer, fieldnotes)

c. Transitive class: jok-t6-um 'to buy'

jok-t6-ek

buy-TR-PRS.EGO.SG

'I am buying (something).' (Widmer, fieldnotes) 


\section{Functional motivation}

\begin{tabular}{ccccccc}
\hline & \multicolumn{2}{c}{ Monovalent } & \multicolumn{2}{c}{ Plurivalent } & \multicolumn{2}{c}{ Total } \\
& $\mathbf{n}$ & $\%$ & $\mathbf{n}$ & $\%$ & $\mathbf{n}$ & $\%$ \\
\hline TR & 0 & 0 & 266 & 100 & 266 & 100 \\
MID & 43 & 82.7 & 9 & 17.3 & 52 & 100 \\
INTR & 124 & 79.5 & 32 & 20.5 & 156 & 100 \\
\hline
\end{tabular}




\section{Functional motivation}

- Intransitive class: mostly monovalent verbs (e.g. el- 'go', dat- 'fall'), few plurivalent verbs that are frequently used with non-referential patient arguments (dza- 'eat', pant- 'spin')

- Middle class: monovalent and plurivalent verbs with "middle semantics" (Kemmer 1993) (e.g. 6it- 'die', dur- 'compete')

- Transitive class: plurivalent verbs (e.g. tup- 'cut', da- 'give', Iwat'forget') 


\section{Comparative perspective}




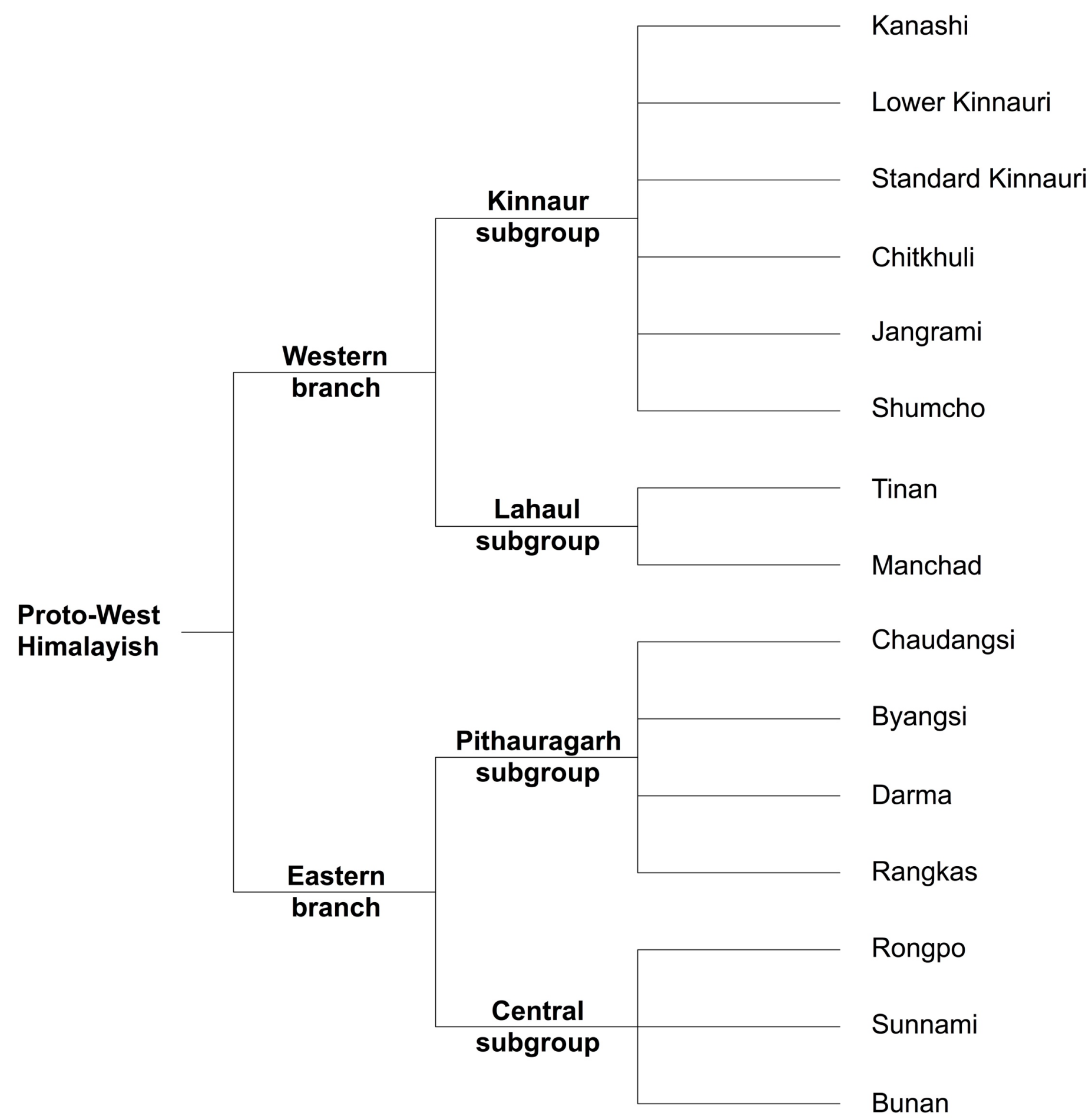




\section{The intransitive class in comparative perspective}

\begin{tabular}{|c|c|c|c|c|}
\hline & Bunan & Rongpo & Darma & Byangsi \\
\hline $1 S G$ & $\begin{array}{l}r a-k \text {-ek } \\
\text { come-INTR-PRS.EGO.SG }\end{array}$ & $\begin{array}{l}\text { bwəl-k-ən் } \\
\text { camp-INTR-PRS.1SG }\end{array}$ & $\begin{array}{l}\text { ra-h-i } \\
\text { come-INTR-PRS.1SG }\end{array}$ & $\begin{array}{l}r a-g-\varepsilon \\
\text { come-INTR-PRS.1SG }\end{array}$ \\
\hline $2 S G$ & $\begin{array}{l}\text { ra-k-ana } \\
\text { come-INTR-PRS.2sG }\end{array}$ & $\begin{array}{l}\text { bwəl-k-ən } \\
\text { camp-INTR-PRS.2sG }\end{array}$ & $\begin{array}{l}\text { ra-h-en } \\
\text { come-INTR-PRS.2sG }\end{array}$ & $\begin{array}{l}\text { ra-g-no } \\
\text { come-INTR-PRS.2sG }\end{array}$ \\
\hline $3 S G$ & $\begin{array}{l}\text { ra-k-are } \\
\text { come-INTR-PRS.ALLO.SG }\end{array}$ & $\begin{array}{l}\text { bwəl-k-ən } \\
\text { camp-INTR-PRS.3sG }\end{array}$ & $\begin{array}{l}\text { ra-ni } \\
\text { come-INTR.PRS.3 }\end{array}$ & $\begin{array}{l}\text { ra-g-an } \\
\text { come-INTR-PRS.3SG }\end{array}$ \\
\hline $1 \mathrm{PL}$ & $\begin{array}{l}\text { gwan-k-hek } \\
\text { come.PL-INTR-PRS.EGO.PL }\end{array}$ & $\begin{array}{l}\text { bwəl-k-əni } \\
\text { camp-INTR-PRS.PL }\end{array}$ & $\begin{array}{l}\text { ra-h-en } \\
\text { come-INTR-PRS.1PL }\end{array}$ & $\begin{array}{l}\text { ra-g-nye } \\
\text { come-INTR-PRS.1PL }\end{array}$ \\
\hline $2 \mathrm{PL}$ & $\begin{array}{l}\text { gwan-k-hakni } \\
\text { come.PL-INTR-PRS.2PL }\end{array}$ & $\begin{array}{l}\text { bwəl-k-əni } \\
\text { camp-INTR-PRS.PL }\end{array}$ & $\begin{array}{l}\text { ra-h-en(i) } \\
\text { come-INTR-PRS.2PL }\end{array}$ & $\begin{array}{l}\text { ra-g-ni } \\
\text { come-INTR-PRS.2PL }\end{array}$ \\
\hline $3 P L$ & $\begin{array}{l}\text { gwan-k-hak } \\
\text { come.PL-INTR-PRS.ALLO.PL }\end{array}$ & $\begin{array}{l}\text { bwəl-k-əni } \\
\text { camp-INTR-PRS.PL }\end{array}$ & $\begin{array}{l}\text { ra-ni } \\
\text { come-INTR.PRS.3 }\end{array}$ & $\begin{array}{l}\text { ra-g-nan } \\
\text { come-INTR-PRS.3PL }\end{array}$ \\
\hline
\end{tabular}




\section{The transitive class in comparative perspective}

\begin{tabular}{|c|c|c|c|c|}
\hline & Bunan & Rongpo & Darma & Byangsi \\
\hline \multirow{2}{*}{$1 S G$} & lik-t6-ek & kaț-c-əウ் & $g a-d-i$ & šun-t-o \\
\hline & do-TR-PRS.EGO.SG & cut-TR-PRS.1SG & do-TR-PRS.1SG & do-TR-PRS.1sG \\
\hline \multirow{2}{*}{$2 s G$} & lik-t6-ana & katt-c-ən & ga-d-en & šun-t-año \\
\hline & do-TR-PRS.2sG & cut-TR-PRS.2SG & do-TR-PRS.2SG & do-TR-PRS.2sG \\
\hline \multirow{2}{*}{$3 S G$} & lik-t6-are & kaț-c-ən & ga-d-a & šun-t-a \\
\hline & do-TR-PRS.ALLO.SG & cut-TR-PRS.3sG & do-TR-PRS. 3 & do-TR-PRS.3sG \\
\hline \multirow{2}{*}{$1 \mathrm{PL}$} & lik-t6-hek & kaț-c-ini & ga-d-en & šun-t-anyye \\
\hline & do-TR-PRS.EGO.PL & cut-TR-PRS.PL & do-TR-PRS.1PL & do-TR-PRS.1PL \\
\hline \multirow{2}{*}{$2 \mathrm{PL}$} & lik-t6-hakni & kaț-c-ini & ga-d-en(i) & šun-t-aṇi \\
\hline & do-TR-PRS.2PL & cut-TR-PRS.PL & do-TR-PRS.2PL & do-TR-PRS.2PL \\
\hline \multirow{2}{*}{ 3PL } & lik-t6-hak & kaț-c-ini & ga-d-a & šun-t-añan \\
\hline & do-TR-PRS.ALLO.PL & cut-TR-PRS.PL & do-TR-PRS. 3 & do-TR-PRS.3sG \\
\hline
\end{tabular}




\section{The middle class in comparative perspective}

\begin{tabular}{|c|c|c|c|c|}
\hline & Bunan & Rongpo & Darma & Byangsi \\
\hline \multirow{3}{*}{ INTR } & el-k-ek & $b w ə l-\boldsymbol{k}-\partial \dot{n}$ & dee-h-i & $r a-g-\varepsilon$ \\
\hline & go-INTR-PRS.EGO.SG & camp-INTR-PRS.1SG & go-INTR-PRS.1SG & come-INTR-PRS.1SG \\
\hline & 'I am going.' & 'I am camping.' & 'I am going.' & 'I am coming.' \\
\hline \multirow{3}{*}{ MID } & su-6-ek & kya:-s-k-ə⿱亠 & $u r-s i-h-i$ & 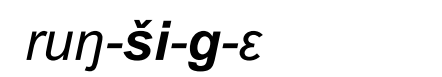 \\
\hline & wash-MID-PRS.EGO.SG & hide-MID-INTR-PRS.1SG & wash-MID-INTR-PRS.1SG & listen-MID-INTR-PRS.1SG \\
\hline & 'I am washing myself.' & 'I am hiding myself.' & 'I am washing myself.' & 'I am listening.' \\
\hline \multirow{3}{*}{ TR } & su-t6-ek & kaț-c-ən் & $u r-\boldsymbol{d}-i$ & šun-t-o \\
\hline & wash-TR-PRS.EGO.SG & cut-TR-PRS.1SG & wash-TR-PRS.1SG & do-TR-PRS.1SG \\
\hline & 'I am washing (so.).' & 'I am cutting (sth.).' & 'I am washing (so.).' & 'I am doing (sth.).' \\
\hline
\end{tabular}

$\begin{array}{lll}\text { Bunan: } & \text { su-6-ek } \\ \text { wash-MID-PRS.EGO } & & \begin{array}{l}{ }^{*} s u-6-k-e k \\ \end{array} \\ & \text { *wash-MID-INTR-PRS.EGO }\end{array}$




\section{Reconstruction}

The reconstructed morphological template of the present tense construction in proto-eastern West Himalayish

\section{VERB STEM - MIDDLE - TRANSITIVITY - TAME / AGREEMENT}




\section{The origins of transitivity markers in EWH}




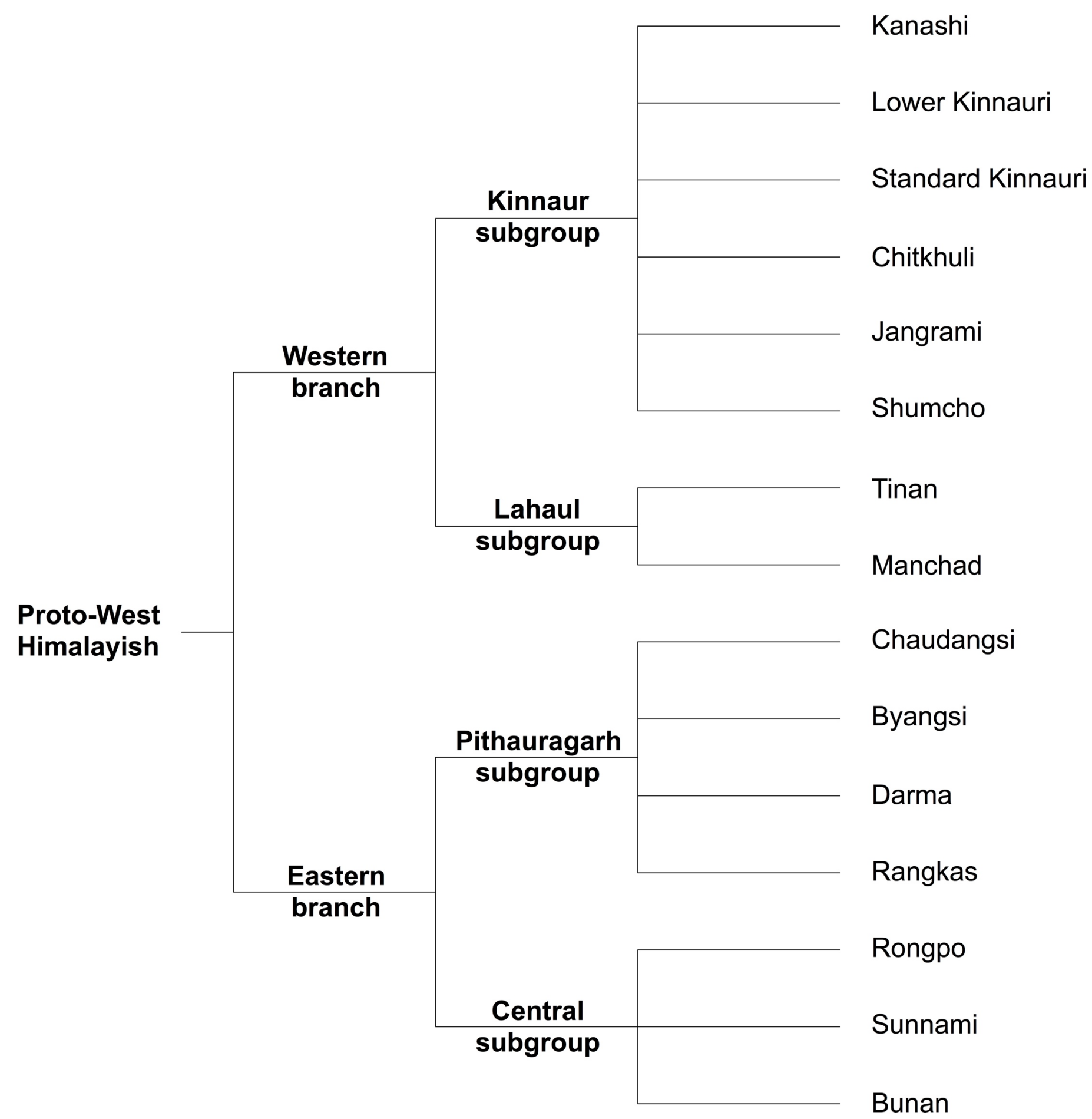




\section{Object agreement markers in Kinnauri}

First / second person object agreement

a. an-t6-o-k

wake.up-1/2O-FUT-1A.SG

'I will wake you up.' (Widmer, fieldnotes)

b. an-t6-o-na

wake.up-1/2O-FUT-2A.SG

'You will wake me up.' (Widmer, fieldnotes) 


\section{Object agreement markers in Kinnauri}

First / second person object agreement

a. an-t6-o-k

wake.up-1/2O-FUT-1A.SG

'I will wake you up.' (Widmer, fieldnotes)

b. an-t6-o-na

wake.up-1/2O-FUT-2A.SG

'You will wake me up.' (Widmer, fieldnotes) 


\section{Universität}

Zürich $^{\text {UzH }}$

Department of Comparative Linguistics

\section{Object agreement markers in Kinnauri}

The Kinnauri object agreement marker -t6- is reminiscent of the Bunan /

Rongpo transitivity marker -t6- both in terms of its function and its morphological position in the verb complex. It is thus probable that the two markers are related and that the transitivity marker developed from an object agreement marker. The question now is: Can we come up with a story that account for this functional reanalysis? 


\section{Transitivity marking in Rawang (LaPolla 2011)}

a. Intransitive class: shì=ē 'die'

$\begin{array}{lllll}\text { ngà rø̀mnv̄ng-pè } & g \bar{\varnothing} & \text { shì } & \text { bǿ-ì } \\ \text { 1SG friend-MALE } & \text { also die } & \text { PFV-INTR.PST } \\ \text { 'My friend also died.' (LaPolla 2011: 637) }\end{array}$

b. Transitive class: $y \dot{n} n g-o ́=\bar{e}$ 'see'

$\begin{array}{lllll}\text { rvshà-rì=i } \quad y \dot{n} n g & \text { bǿ-à } & k v t & \ldots \\ \text { monkey-PL=AGT } \quad \text { see } & \text { PFV-TR.PST } & \text { when } & \ldots \\ \text { 'When the monkeys saw (him), ... .' (LaPolla 2011: } & \text { 638) }\end{array}$




\section{Transitivity marking in Rawang (LaPolla 2011)}

- Depending on the grammatical context, the marker -ó- either functions as an $3^{\text {rd }}$ person object agreement marker or a marker of transitive predicates.

- This makes it plausible that there is a diachronic link between the functional domains of object marking and transitivity marking! But how could this reanalysis be explained? 


\section{The reanalysis}

Object agreement markers can sometimes occur in contexts in which they do not serve the function of indexing to a specific object argument (e.g. in citation forms as is the case in Rawang). This opens up the possibility of a metanalysis (Croft 2001: 130), viz. the swapping of «contextual and inherent semantic values of a syntactic unit». 


\section{Universität}

Zürich $^{\mathrm{UzH}}$

Department of Comparative Linguistics

This scenario offers a plausible explanation for the origin of the transitive marker in EWH languages, but where does the intransitive marker come from? It is possible that this morpheme is the reflex of an old converb marker *-ka, which ended up inside of an inflected form due to the univerbation of a formerly periphrastic verb form. 
Monovalent verbs

\section{Stage 1}

Stage 2

Stage 3

Stage 4
${ }^{*} V-k-S U B J$

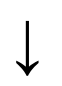

${ }^{*} V-k a+C O P-S U B J$

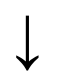

${ }^{*} V-k-S U B J$

$\downarrow$

$V-K$-SUBJ

${ }^{*} V-\boldsymbol{k}$-SUBJ

\section{Plurivalent verbs}

${ }^{*} V\left(-t_{6}\right)-k a+C O P-S U B J$

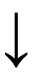

${ }^{*} V(-t 6)-k-S U B J$

$\downarrow$

${ }^{*} V-t 6-k-S U B J$

$\downarrow$

${ }^{*} V$-t6-SUBJ 


\section{References}

Dixon, Robert M. W. 2010. Basic linguistic theory, volume II: grammatical topics. Oxford: Oxford University Press.

Hopper, Paul J. \& Sandra A. Thompson. 1980. Transitivity in grammar and discourse. Language 56(2), 251-299.

LaPolla, Randy J. 2011. On transitivity in two Tibeto-Burman languages. Studies in Language 35(3), 636-649.

Widmer, Manuel. 2017. A grammar of Bunan (Mouton Grammar Library 71). Berlin: Mouton de Gruyter.

Widmer, Manuel. 2018. Transitivity markers in West Himalayish: synchronic and diachronic considerations. Linguistics of the Tibeto-Burman Area 41(1), 75-105. 